\title{
Adaptive Equalization of WACS in Time-Dispersive, Fading, Multipath Channels
}

\author{
Gary Boray and Prem Sood \\ Sharp Microelectronics Technology \\ 5700 NW Pacific Rim Blvd., Camas, WA 98607
}

\begin{abstract}
WACS (Wireless Access Communications Systems) developed by Bellcore is the basis of a proposed PCS standard in the $2 \mathrm{GHz}$ emerging technology band in United States. WACS is ideally suited for indoor applications. It is generally known that operation in outdoor, large cell environments will require suitable receiver techniques to combat the effects of time-dispersive (delay-spread), fading, multipath channels. In this paper, we study the effectiveness of down-link adaptive equalization for improving the performance of WACS for outdoor applications. A discussion on Decision Feedback Equalization with different adaptation algorithms such as Least Mean Squares (LMS) methods and Recursive Least Squares (RLS) methods are provided. Joint Technical Committee (JTC) of Committee T1 and TIA in United States has developed comprehensive $\mathrm{RF}$ channel models to represent typical indoor and outdoor channel conditions. Simulation results of applying adaptive equalization to WACS with the JTC outdoor RF channel models are presented. The results indicate that with a judicious choice of adaptation algorithm, it is possible to obtain adequate performance in outdoor channels while remaining within the bounds of reasonable complexity.
\end{abstract}

\section{INTRODUCTION}

The WACS (Wireless Access Communications Systems) PCS system, originally proposed by Bellcore is the basis of an evolving PCS standard called PACS (Personal Access Communications System) in the $2 \mathrm{GHz}$ band in United States. WACS has a simple radio architecture compared to other advanced TDMA schemes such as IS-54, GSM, etc. For example, WACS does not incorporate elaborate error control schemes such as convolutional coding. It has a simple error detection scheme. The simple radio architecture proposed by WACS will suffice for indoor applications, where the channel conditions are not as hostile as in large cell, outdoor, vehicular conditions. In this paper, we report on a study undertaken to extend WACS for large cell, outdoor, vehicular applications. This will extend the coverage capabilities of WACS and would result in a single handset for indoor and outdoor applications. Further, enlarged coverage translates to fewer cell sites, which will result in lowered infrastructure costs. Large cell, outdoor, vehicular conditions result in fast fading multipath channels with significant delay spreads. There are several approaches to combat these hostile channels. Some of the approaches are - frequency hopping, adaptive equalization, convolutional coding, diversity reception etc. Among the many approaches the ones that do not require modifications to the standard air-interface has obvious advantages. With this view, we performed a study of down-link adaptive equalization of WACS for outdoor applications. The rest of the paper is organized as follows. In section II, we provide an outline of the WACS system and the simulation setup. Section III deals with the design of adaptive equalizers. In section IV, we provide results of simulations with fading, multipath channels. The Joint Technical Committee (JTC) of TIA and Committee T1 in N. America has developed comprehensive propagation models to represent the various indoor and outdoor environments. We provide simulation results of applying adaptive equalization to WACS with some typical, outdoor, vehicular JTC models. The paper is concluded in section $\mathrm{V}$.

\section{SuMmaRy OF WACS SYSTEM CONFIGURATION}

\section{A. WACS System Configuration}

The WACS PCS system [4] is a TDMA/FDD system with the following system parameters:

\author{
- $\pi / 4$ shifted DQPSK Modulation \\ - Bandwidth $=300 \mathrm{kHz}$ \\ - Bit Rate $=400 \mathrm{Kbps}$ \\ - Symbol Rate $=200 \mathrm{Ksymbols} / \mathrm{sec}$ \\ - \# Time slots/Frame= 8 \\ - Slot Duration $=250$ microsecs (i.e., 50 symbols)
}

\section{B. Slot Structure}

\begin{tabular}{|c|c|c|c|c|}
\hline $\begin{array}{l}\text { Sync } \\
14 \\
\text { bits }\end{array}$ & $\begin{array}{l}\text { Slow } \\
\text { Signal } \\
9 \text { bits }\end{array}$ & $\begin{array}{l}\text { Info. } \\
64 \\
\text { bits }\end{array}$ & $\begin{array}{l}\text { Error } \\
\text { Detect } \\
12 \text { Bits }\end{array}$ & $\begin{array}{l}\text { Power } \\
\text { Control } \\
1 \text { bit }\end{array}$ \\
\hline
\end{tabular}

Fig. 1. Slot Structure of WACS Down-Link Channels

Fig. 1 depicts the slot structure for a WACS down-link channel. Among the 23 bits allocated for synchronization and slow associated signaling as shown in Fig. 1, we propose the use of the 16 known bits for training the equalizer. 


\section{Simulation Set- $U p$}

Fig. 2 is a link level simulation model for WACS which incorporates the DQPSK modulator, raised cosine transmit and receive filters, Rayleigh faded, delay-spread, multipath channel and the additive Gaussian noise. Coherent symbol detection was performed in the receiver. Finally, the detected symbols were differentially decoded to extract the output bits.

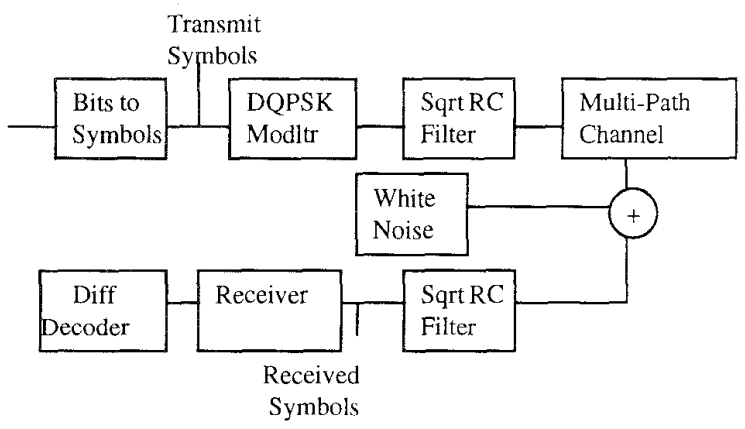

Fig. 2. System Model for Generation of WACS Transmit and Receiver Symbols

\section{ADAPTIVE EQUALIZERS FOR WACS}

The two main classes of equalizers are - linear equalizers and non-linear equalizers. An example of a linear equalizer is a transversal filter, whose output is computed as the weighted sum of delayed replicas of the input. The most widely used non-linear equalizer is the Decision Feedback Equalizer (DFE), which has two transversal filter sections: a feed forward section and a feedback section. In the feedback section, previously detected, known decisions are fed back. It is well known, that for highly distorted channels, one has to resort to non-linear equalization to obtain acceptable performance [12]. The advantage of DFE is that, by feeding back known, previously detected symbols, one can remove that part of the intersymbol interference from the present estimate caused by previously detected symbols. In this paper, we have used a decision feedback equalizer. Further, typically the taps in the feed forward transversal filter of a DFE are spaced $T / 2$, where $T$ is the symbol duration, while in the feedback section the taps are symbol spaced. The use of fractional spacing in the feed forward filter, provides some resistance to errors that may occur due to timing inaccuracies. Adaptive algorithms are typically used to update the taps of a DFE. In this paper, we will consider the two most widely used adaptive algorithms, namely, LMS (Least Mean Squares) algorithm and RLS (Recursive Least Squares) algorithm.

\section{A. Complex LMS Equalizer}

The LMS algorithm is the simplest and the most widely used adaptation algorithm. The coefficients of the feed forward and the feedback filter in a DFE that employs complex LMS algorithm [8] are updated according to the following adaptation equations.

$y=W_{f}^{\prime} X+W_{b}^{\prime} D$

$W_{f}=W_{f}+\mu \quad(d-y) X^{*}$

$W_{b}=W_{b}+\mu \quad(d-y) D^{*}$

In these equations:

- $y$ is the unquantized equalizer output

- $X$ is the vector of feed forward filter inputs

- $W_{f}$ is the vector of $m_{f}$ feed forward complex filter weights

- $W_{b}$ is the vector of $m_{b}$ feedback complex filter weights

- $d$ is the desired signal

- $\quad D$ is the vector of past $d$ values

- $\mu$ is the adaptive step size

- $m=m_{b}+m_{f}$

- $W=\left[\begin{array}{lll}W_{b} & W_{f}\end{array}\right]$ is the vector of forward and backward weights

- The symbol * denotes complex conjugation, while the prime (') denotes complex-conjugate transposition.

The weight updates are performed only once per symbol, ideally at the symbol center. This is the point at which the eye is most open. The desired response $d$ is a training sequence during the training mode or the slicer decisions during the data mode (decision directed mode).

\section{B. Complex RLS Equalizer}

The RLS (Recursive Least Squares) algorithm is a deterministic version of the classical Kalman Filter algorithm [3]. An important feature of the RLS algorithm is that it utilizes all the information contained in the input data, extending back to the time when the algorithm is initiated. The resulting rate of convergence is therefore typically an order of magnitude faster than the simple LMS algorithm. This improvement in performance, however, is achieved at the expense of a large increase in computational complexity.

The following steps are implemented in the RLS algorithm, once every symbol.

$x=\lambda^{-1} P U$ 
$k=x /\left(1+U^{\prime} x\right)$

$e=d-W^{\prime} U$

$W=W+k e^{*}$

$P=\lambda^{-l} P-k x^{\prime}$

In these equations,

- $\quad P$, the inverse correlation matrix is of dimension $(m \times$ $m$ ) and is initialized by setting $P=\delta{ }^{-I} I$, where, $I$ is an $(m \times m)$ Identity matrix, and, $\delta$ is a small positive constant.

- $U=\left[\begin{array}{ll}X & D\end{array}\right]$, is the $m$ dimensional vector composed of inputs to the feed forward and feed back filter sections.

- $\lambda$ is the so-called forgetting factor, which is typically close to, but, less than 1 .

RLS algorithms provide a faster convergence compared to LMS. They have a fixed computational complexity of the order $\mathrm{O}\left(\mathrm{m}^{2}\right)$. Some modifications in reducing the complexity of RLS algorithm have resulted in a class of algorithms called fast RLS algorithms [6,7].

\section{Performance Of LMS ANd RLS DFE In Time- Dispersive, Fading, Multipath Channels}

Simulations were performed with the set-up described in section 2, to verify the performance of LMS and RLS Decision Feedback Equalizers for fading, delay-spread, multipath channels in the presence of additive white Gaussian noise. First, we employed a standard 2-ray channel model to verify the performance of DFE. Then, we used outdoor, vehicular models specified by JTC to study the impact of equalization on WACS.

\section{A. Performance of LMS and RLS DFE with 2-Ray Channel Model}

A standard 2-ray model was used, with each ray being of equal average power. The 2 rays are separated by a delay, commonly known as the delay spread. The normalized delay spread parameter, $\tau$, is expressed as a ratio of the delay spread to the symbol duration. In our simulations, we have used values of $\tau$ from 0.125 to 0.75 , to simulate a wide range of delay spread conditions representing very small cells to relatively large cells. Vehicular motion results in Doppler shift, which is represented by the Doppler frequency parameter $f_{d}$.
Simulations were performed for a high delay spread case of $\tau=0.75$, at signal-to-noise ratio $(\mathrm{Eb} / \mathrm{N} 0)$ of $21 \mathrm{~dB}$ and Doppler frequency of $5 \mathrm{~Hz}$. DFE(4,1) was employed with 4 feed-forward taps and 1 feedback tap. Mean squared error in the equalizer output averaged over 1000 slots are plotted as a function of the processed symbol in Fig. 3. Simulations were repeated at a higher Doppler of $50 \mathrm{~Hz}$ and plotted in Fig. 4. Results indicate that RLS provides faster convergence compared to LMS. Further at high Doppler speeds, as seen in Fig. 4, LMS tends to diverge.

Fig. 5 is a plot of BER v/s Eb/N0 for LMS and RLS $\operatorname{DFE}(4,1)$ at $\tau=0.75$ and Doppler of $5 \mathrm{~Hz}$. Results indicate that the RLS provides much better performance compared to the LMS equalizer. This is due to the superior convergence properties of the RLS adaptive algorithm.

Fig. 6 is a plot of BER v/s delay spread for LMS and RLS DFE(4,1) at Eb/NO $=21 \mathrm{~dB}$ and Doppler $=5 \mathrm{~Hz}$. Results again indicate that RLS equalizer is able to provide acceptable bit-error-rate performance.

\section{B. Performance of LMS and RLS DFE with JTC Outdoor Channel Models}

In this section we provide simulation results for LMS and RLS decision feedback equalizers with JTC vehicular, outdoor models. A draft document form JTC [2] has recommended the following 9 different classes of channels to represent the various indoor and outdoor conditions that will be encountered by typical PCS systems.

(i) Indoor Residential

(ii) Indoor Office

(iii) Indoor Commercial

(iv) Outdoor Pedestrian Urban High-Rise

(v) Outdoor Pedestrian Urban/Suburban Low Rise

(vi) Outdoor Pedestrian Residential

(vii) Outdoor Vehicular Urban High-Rise

(viii) Outdoor Vehicular Urban/Suburban Low-Rise

(ix) Outdoor Vehicular Residential

In order to incorporate the large amount of variability of delay spread within a given environment three multipath channels are defined for each environment. Channel $A$ is the low delay spread case that occurs frequently, channel $B$ is the median delay spread case that also occurs frequently, and channel $\mathrm{C}$ is the high delay spread case that occurs only rarely. The outdoor vehicular models (vii), (viii) and (ix) present the most hostile conditions as they represent delay spread multipath channels with very fast fading due to the vehicular motion. We performed simulations with the JTC channel models $7 \mathrm{~B}, 8 \mathrm{~B}$ and $9 \mathrm{~B}$ which corresponds to 
outdoor, vehicular models (vii), (viii), and (ix) with median delay spread. The ratio of RMS delay spread to the symbol duration for models $7 \mathrm{~B}, 8 \mathrm{~B}$, and, $9 \mathrm{~B}$ are $0.65,0.80$, and, 0.45 respectively. Fig. 7a, Fig. $7 \mathrm{~b}$ and Fig. $7 \mathrm{c}$ depict the power delay profiles for the JTC channel models JTC 7B, $8 \mathrm{~B}$ and $9 \mathrm{~B}$. Channels $7 \mathrm{~B}$ and $9 \mathrm{~B}$ were simulated with a Doppler frequency of $50 \mathrm{~Hz}$, corresponding to vehicular speed of around $20 \mathrm{mph}$, while channel $8 \mathrm{~B}$ was simulated with a Doppler frequency of $100 \mathrm{~Hz}$, which corresponded to a vehicular speed of around $40 \mathrm{mph}$. Simulations were performed at a signal-noise-ratio $\mathrm{E}_{\mathrm{b}} / \mathrm{N}_{0}=21 \mathrm{~dB}$. DFE with 6 feed forward and 1 feedback tap was utilized in the simulations. Table I lists the bit-error-rates for the 3 JTC channel models for both LMS and RLS equalizers. Results for the case of 'No Equalizer' corresponds to the use of differential detector only in the receiver.

TABLE I

Bit Error Rates For JTC ChanNel Models

\begin{tabular}{|l|l|l|l|}
\hline Equalizer\Channel & JTC 7B & JTC 8B & JTC 9B \\
\hline No Equalizer & 0.1104 & 0.1600 & 0.2113 \\
\hline LMS DFE $(6,1)$ & 0.2040 & 0.4422 & 0.3371 \\
\hline RLS DFE $(6,1)$ & 0.0329 & 0.0336 & 0.0037 \\
\hline
\end{tabular}

Results from Table $I$ indicate that the LMS equalizer is unable to equalize the outdoor vehicular channels. This is principally due to the slow convergence of the LMS adaptive algorithm, Further, the bit-error-rate for the LMS equalizer exceeds the bit-error-rate without equalizer. This is due to the use of decision feedback equalizer, where frequently occurring, incorrect past decisions get fed back resulting in increased errors. RLS equalizers provide much lower bit-error-rate approaching $3 \%$.

\section{CONCLUSIONS}

Extension of WACS for outdoor applications has many advantages. However, to do so requires that adequate measures be taken to assure acceptable performance in severely distorted multipath, fading channels. Decision feedback equalization is a viable technique that can be employed at the mobile receiver to enable outdoor operation. Further, equalization can be implemented in mobile terminals without any explicit changes to the airinterface specifications. Simulations reveal that a simple adaptive algorithm such as LMS is unable to equalize the outdoor channels specified by JTC. One can obtain reasonable performance with a robust algorithm such as RLS. It is recommended that low complexity implementations of RLS such as fast RLS, fast Kalman etc., $[3,6,7,9]$ be investigated to retain the performance of RLS at much lower complexity. It is also noted that since the downlink in the WACS system is continuously transmitting (i.e., TDM mode), it is possible to utilize the synchronization sequences of adjacent slots, and, thereby effectively providing additional opportunities for retraining the equalizer [5]. This will result in enhanced performance.

\section{REFERENCES}

[1] J. Fuhl, G. Schultes, W. Kozek, "Adaptive equalization for DECT systems operating in low time-dispersive Channels", IEEE Vehicular Technology Conference, 1994.

[2] Deployment and Testing Ad Hoc Group, "Draft Technical Report on RF Channel Characterization and System Deployment Modeling", JTC (AIR)/94.08.01-065R14, August 1994

[3] Simon Haykin, "Adaptive Filter Theory", Prentice-Hall Publishers

[4] PPS-1800 (TM) - Local Service Common Air Interface Proposal, JTC(AIR)/93.11.01.420, Motorola, Inc.

[5] Y.J. Liu, M. Wallace, J.W. Ketchum, " A soft-output bi-directional decision feedback equalization technique for TDMA cellular radio", IEEE J. Select. Areas Commun., vol. 11, no. 7, Sept. 1993.

[6] M. Morf, T. Kailath, and L. Ljung, "Fast algorithms for recursive identification", Proc. 1976 Conf. Decision and Control, Clearwater Beach, FL.

[7] J.M. Cioffi and T. Kailath, "Fast, recursive-least-squares transversal filters for adaptive filtering", IEEE Trans. Acoust., Speech, and Signal Processing, vol. 32.

[8] B. Widrow, J. McCool, and M. Ball, "The complex LMS algorithm", Proceedings of the IEEE, April 1975.

[9] F. Hsu, "Square root Kalman filtering for high-speed data received over fading dispersive HF channels", IEEE Trans. Inform. Theory, vol. 28, No. 5, Sept. 1982.

[10] TA-NWT-01313, Generic Criteria for Version 0.1 Wireless Access Communication Systems (WACS), Bellcore, Issue 1, October 1993.

[11] D.C. Cox, "A radio system proposal for widespread low-power tetherless communications", IEEE Trans. Commun., vol. 39, pp. 324-334, Feb. 1991.

[12] J. G. Proakis, Digital Communications, McGraw-Hill, 1989.

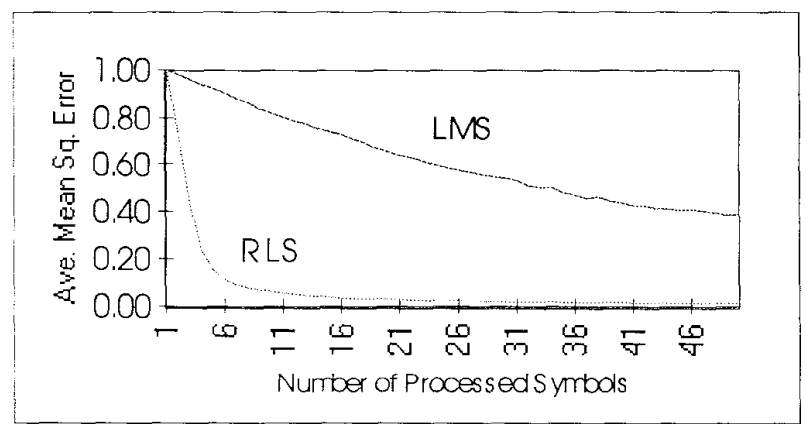

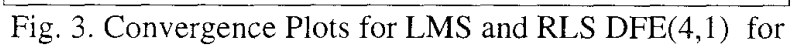
$\tau=0.75, \mathrm{fd}=5 \mathrm{~Hz}, \mathrm{~Eb} / \mathrm{NO}=21 \mathrm{~dB}$ 


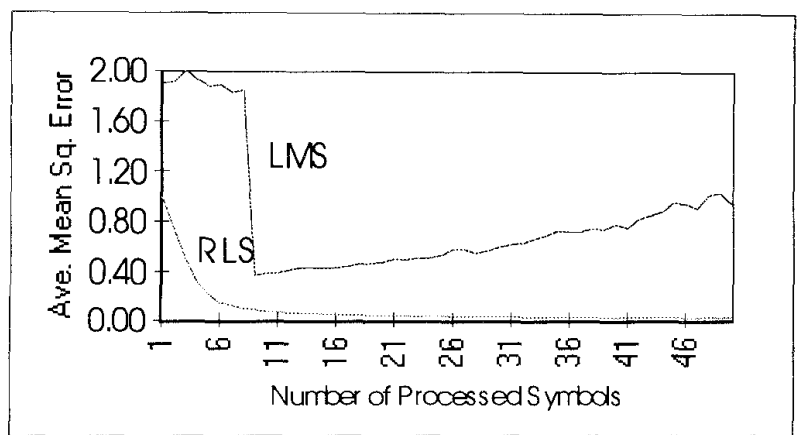

Fig. 4. Convergence Plots for LMS and RLS DFE(4,1) for $\tau=0.75, \mathrm{fd}=50 \mathrm{~Hz}, \mathrm{~Eb} / \mathrm{N} 0=21 \mathrm{~dB}$

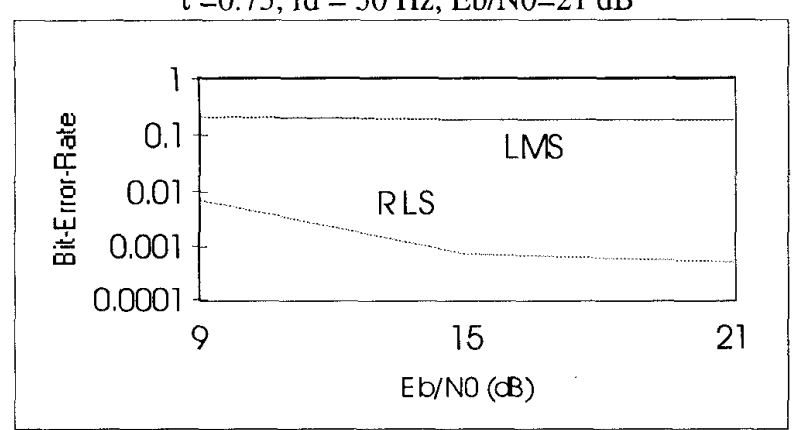

Fig. 5. BER v/s Eb/N0 for LMS and RLS for $\tau=0.75$, $\mathrm{fd}=5 \mathrm{~Hz}$

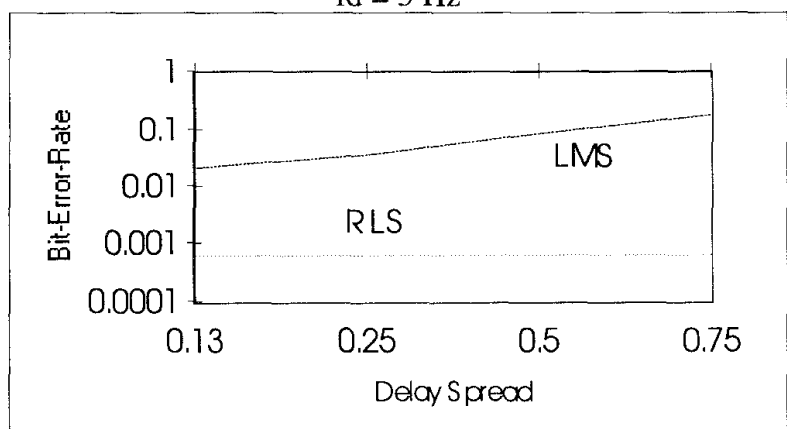

Fig. 6. BER v/s $\tau$ for LMS and RLS for $\mathrm{Eb} / \mathrm{NO}=21 \mathrm{~dB}$, $\mathrm{fd}=5 \mathrm{~Hz}$

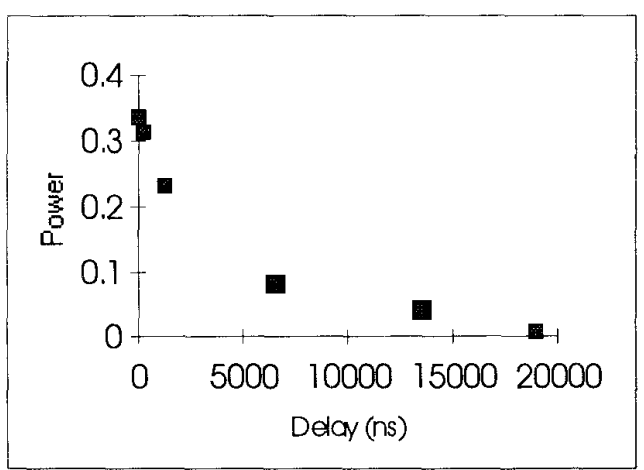

Fig. 7a. Power Delay Profiles for JTC Channel Model JTC 7B

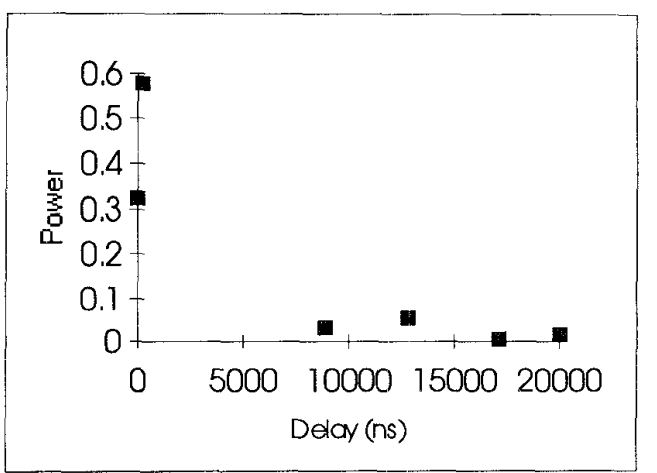

Fig. 7b. Power Delay Profiles for JTC Channel Model JTC 8B

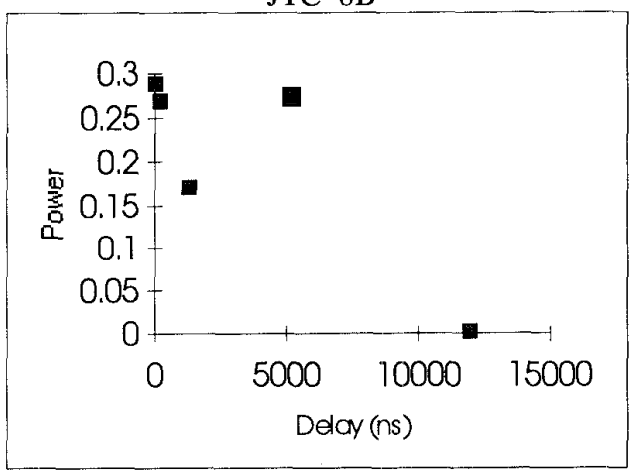

Fig. 7c. Power Delay Profiles for JTC Channel Model JTC 9B 